J. Austral. Math. Soc. 20 (Series A) (1975), 77-84.

\title{
AUTOMORPHISMS OF TRANSFORMATION SEMIGROUPS
}

\author{
R. P. SULLIVAN \\ (Received 16 July 1973) \\ Communicated by G. B. Preston
}

\section{Introduction}

We let $X$ be an arbitrary non-empty set throughout. Many papers have been written describing the automorphisms of various transformation semigroups defined on $X$ : total (Lyapin (1955), Magill (1967), Malcev (1952), Schreier (1936)), partial (Gluskin (1959), Magill (1967)), partial and 1-1 (Liber (1953)), partial and shifting at most a finite number of elements (Subov (1961a)). In all these cases the automoprhisms are shown to be "inner", and using the fact this authors deduce that the automorphism group of the given transformation semigroup is isomorphic to the group $\mathscr{G}_{X}$ of all permutations defined on $X$.

In theorems 1 and 2 below we provide a simple generalization of this work, and in $\S 3$ indicate the way in which they may be used to obtain each of the abovementioned results, together with new ones of some independent interest.

The work for this paper formed part of Sullivan (1969) written under the supervision of Professor G. B. Preston; we here gratefully acknowledge his assistance and encouragement.

\section{Semigroups covering $X$.}

Our notation will be that of Clifford and Preston $(1961,1967)$ and Scott (1964) unless otherwise specified. As in Magill (1967) we let $A_{x}$ denote the element of $\mathscr{P}_{X}$ with domain $A$ and range $x$ and call it a constant based on $x$, and as in Scott (1964) a 2-cycle in $\mathscr{G}_{X}$ will be denoted by $(x, y)$. A subsemigroup $S$ of $\mathscr{B}_{X}$ is said to cover $X$ if for every $x \in X$, there exists some constant idempotent in $S$ based on $x$ and $S$ is said to be $\mathscr{G}_{X}$-normal if for all $\alpha \in S, g \in \mathscr{G}_{X}, g^{-1} \alpha g \in S$.

LEMMA 1. If $S$ is a $\mathscr{G}_{X}$-normal subsemigroup of $\mathscr{B}_{X}$ containing a constant based on some point of $X$, then $S$ covers $X$.

Proof. Suppose $A_{z} \in S$ with $z \notin A$, and let $a \in A$. We then obtain $(z \cup A \backslash a)_{a}$ $=(z, a) \cdot A_{z} \cdot(z, a) \in S$, and so $\left((z \cup A \backslash a)_{z}=(z \cup A \backslash a)_{a} \cdot A_{z} \in S\right.$ and is a con- 
stant idempotent based on $z$. Suppose $D=z \cup A \mid a$ and let $x \in X, x \neq z$. Then the mapping $\alpha=(x, z) \cdot D_{z} \cdot(x, z)$ is a constant in $S$ based on $x$ and $\alpha^{2}=\alpha$. The converse of the above lemma is not true:

EXAmple 1. Let $N$ be the set of positive integers and for each $m, n \in N$, put $B_{m}=\{1, \cdots, m\}$ and $\alpha_{m n}=B_{m} \times n$, the constant mapping based at $n$ with domain $B_{m}$. Then $S=\left\{\alpha_{m n}: m, n \in N\right\} \cup \square$ is in fact a subsemigroup of $\mathscr{P}_{N}$ in which $\alpha_{m n} \alpha_{p q} \neq \square$ if and only if $n \in B_{p}$, and in this case $\alpha_{m n} \alpha_{p q}=\alpha_{m q}$. Clearly $S$ covers $N$. But $S$ is not $\mathscr{G}_{N}$-normal since for example $(2,3) \alpha_{22}(2,3)=\{1,3\}_{3} \notin S$.

Since the semigroup constructed above provides the basis for further counter-examples (see below) we now assume the notation introduced above without further mention. We also let $\operatorname{Aut}(S)$ denote the set of all automorphisms of the subsemigroup $S$ of $\mathscr{B}_{X}$ and $K(S)$ the set of all constant mappings in $S$ with $\square$ adjoined. Then under composition $\operatorname{Aut}(S)$ is a group and $K(S)$ is a semigroup Note that in general the set of all constant idempotents in $S$ will not form a subsemigroup of $S$, even if $\square$ is adjoined: both $\alpha_{32}$ and $\alpha_{54}$ are idempotent, but $\alpha_{32} \alpha_{54}=\alpha_{34}$ is not. Finally, if $\alpha \in \mathscr{T}_{X}$ and $A \subseteq X, A \neq \square$, we let $\alpha \mid$ A denote the restriction of $\alpha$ to $A$. We now have

LemMa 2. If $S$ is a subsemigroup of $\mathscr{P}_{X}$ covering $X$ and if $\phi \in \operatorname{Aut}(S)$, then $\phi \mid K(S) \in \operatorname{Aut}(K(S))$.

Proof. We first show that $\phi$ maps any constant idempotent of $S$ to another. To this end let $\alpha \in X, A_{x} \in K(S), x \in A$, and $A_{x} \phi=\alpha$. Then $\alpha^{2}=\alpha$ and there exists $z \in X \alpha$ such that $z \alpha=z$. Since $S$ covers $X$, we may choose $B_{z} \in K(S)$ with $z \in B$. Letting $\lambda \phi=B_{z}$, we then have $\lambda^{2}=\lambda$ and $\left(\lambda A_{x}\right) \phi=B_{z} \alpha=B_{z}=\lambda \phi$. Hence $\lambda=C_{x}$ for some $C \subseteq X$ with $x \in C$, and so $\alpha=A_{x} \phi=\left(A_{x} C_{x}\right) \phi=\alpha B_{z}$. Hence $\alpha=D_{z}$ for some $D \subseteq X$ and $z \in D$, and our assertion holds.

Now since $\phi \in \operatorname{Aut}(S)$, we will have $\square \phi=\square \in K(S)$, and if $A_{x} \in K(S)$ with $x \notin A$, then there exists $B_{x} \in K(S)$ with $x \in B$, and from above $B_{x} \phi=C_{z}$ for some $C \subseteq X$ with $z \in C$. Hence letting $A_{x} \phi=\alpha$, we obtain $\alpha^{2}=\square$ and $\alpha=A_{x} \phi=\left(A_{x} B_{x}\right) \phi=\alpha C_{z}$; that is, $\alpha=D_{z}$ for some $D \subseteq X$ with $z \notin D$

We have therefore shown that $\phi$ maps $K(S)$ into $K(S)$. Since $\phi^{-1} \in \operatorname{Aut}(S)$, $\phi^{-1}$ will do the same and hence $\phi \mid K(S)$ is surjective, and the result follows.

The converse of the above lemma is clearly not true, since for example if $T=\left\{\alpha_{m n}: n \neq 1\right\} \cup \square$, then $T$ is a subsemigroup of $\mathscr{P}_{N}$ which does not cover $N$, but for which $K(T)=T$.

It is quite difficult to determine a simple criterion in order that a subsemigroup $S$ of $\mathscr{B}_{X}$ should have the property: $\phi \mid K(S) \in \operatorname{Aut}(K(S))$ for all $\phi \in \operatorname{Aut}(S)$ (see Sullivan (to appear)). It is not true for example that the above Lemma can be extended to subsemigroup of $\mathscr{B}_{X}$.

ExAmple 2. Put $\beta_{m n}=\beta_{m} \times\{n, n+1\}$ for each $m, n \in N$, and let $S=\left\{\alpha_{m n}: m, n \in N\right\} \cup\left\{\beta_{m n}: m, n \in N\right\} \cup \square$. Then $S$ is a subsemigroup of $\mathscr{B}_{N}$ 
in which for example $\beta_{m n} \cdot \beta_{p q} \neq \square$ if and only if $n \in B_{p}$, and in this case $\beta_{m n} \cdot \beta_{p q}$ $=\beta_{m q}$. Clearly $S$ covers $N$. But we assert that the mapping $\phi: S \rightarrow S$ defined by setting $\alpha_{m n} \phi=\beta_{m n}, \beta_{m n} \phi=\alpha_{m n}$, and $\square \phi=\square$, is an automorphism of $S$ for which $\phi \mid K(S) \notin \operatorname{Aut}(K(S))$. For, if $n \leqq p$, we have

$$
\begin{aligned}
& \left(\alpha_{m n} \cdot \alpha_{p q}\right) \phi=\left(\alpha_{m q}\right) \phi=\beta_{m q}=\beta_{m n} \cdot \beta_{p q}=\alpha_{m n} \phi \cdot \alpha_{p q} \phi \\
& \left(\alpha_{m n} \cdot \beta_{p q}\right) \phi=\left(\beta_{m q}\right) \phi=\alpha_{m q}=\beta_{m n} \cdot \alpha_{p q}=\alpha_{m n} \phi \cdot \beta_{p q} \phi \\
& \left(\beta_{m n} \cdot \alpha_{p q}\right) \phi=\left(\alpha_{m q}\right) \phi=\beta_{m q}=\alpha_{m n} \cdot \beta_{p q}=\beta_{m n} \phi \cdot \alpha_{p q} \phi \\
& \left(\beta_{m n} \cdot \beta_{p q}\right) \phi=\left(\beta_{m q}\right) \phi=\alpha_{m q}=\alpha_{m n} \cdot \alpha_{p q}=\beta_{m n} \phi \cdot \beta_{p q} \phi
\end{aligned}
$$

and if $p<n<n+1$, we have

$$
\begin{aligned}
& \left(\alpha_{m n} \cdot \alpha_{p q}\right) \phi=\square=\beta_{m n} \cdot \beta_{p q}=\alpha_{m n} \phi \cdot \alpha_{p q} \phi \\
& \left(\alpha_{m n} \cdot \beta_{p q}\right) \phi=\square=\beta_{m n} \cdot \alpha_{p q}=\alpha_{m n} \phi \cdot \beta_{p q} \phi \\
& \left(\beta_{m n} \cdot \alpha_{p q}\right) \phi=\square=\alpha_{m n} \cdot \beta_{p q}=\beta_{m n} \dot{\phi} \cdot \alpha_{p q} \phi \\
& \left(\beta_{m n} \cdot \beta_{p q}\right) \phi=\square=\alpha_{m n} \cdot \alpha_{p q}=\beta_{m n} \phi \cdot \beta_{p q} \phi
\end{aligned}
$$

Moreover, if $\alpha_{m n} \phi=\alpha_{p q} \phi$, then $B_{m}=B_{p}$ and $\{n, n+1\}=\{q, q+1\}$ together imply that $m=p$ and $n=q$, and so $\phi$ is injective. Since $\phi$ is clearly surjective we conclude that $\phi \in \operatorname{Aut}(T)$, and the assertion follows.

If $S$ is a subsemigroup of $\mathscr{B}_{X}$ and $\phi \in \operatorname{Aut}(S)$, then $\phi$ is inner if there exists $g \in \mathscr{G}_{X}$ such that $\alpha \phi=g^{-1} \alpha g$ for all $\alpha \in S$; all other automorphisms of $S$ will be called outer. It is readily seen that the automorphism constructed in Example 2 is outer: in that case the semigroup used covered $X$ but was not a transformation semigroup; we shall prove below that the automorphisms of the subsemigroup of $\mathscr{P}_{X}$ covering $X$ are always inner. To this end, we next prove:

LEMMA 3. If $S$ is a subsemigroup of $\mathscr{B}_{X}$ covering $X$, then every automorphism of $K(S)$ is inner.

Proof. Let $\phi \in \operatorname{Aut}(K(S))$ and $x \in X$. Since $S$ covers $X$, we may choose $A_{x} \in K(S)$ with $x \in A$. Define $g \in \mathscr{B}_{X}$ by

$$
x g=y \text { if and only if } A_{x} \phi=B_{y}
$$

We assert that $g \in \mathscr{G}_{X}$. For suppose $A_{x} \phi=B_{y}$. If there exists $C_{x} \in K(S)$ with $x \in C$ and $C_{x} \phi=D_{z}$, then $B_{y}=\left(A_{x} C_{x}\right) \phi=B_{y} \cdot D_{z}$, and hence $y=z$; that is, $g$ is a well-defined mapping from $X$ to $X$. In addition, if $x g=y=z g$, then $A_{x} \phi=B_{y}$ and $C_{z} \phi=D_{y}$ where $x \in A$ and $z \in C$, and so $y \in D$ and $B_{y}=B_{y} \cdot D_{y}=\left(A_{x} C_{z}\right) \dot{\phi}$. Hence $A_{x}=A_{x} \cdot C_{z}$ and so $x=z$; that is $g$ is injective. Finally, since $\phi$ is surjective, there exists $D_{z} \in K(S)$ such that $D_{z} \phi=A_{x}$. But then $z \in D$ since $x \in A$, and so $z g=x$; that is, $g$ is surjective. 
Now, if $A_{x} \phi=B_{y}$ and $a \in A$, then there exists $C_{a} \in K(S)$ with $a \in C$ and we have $C_{a} \phi=D_{a g}$. Hence $C_{x} \phi=\left(C_{a} \cdot A_{x}\right) \phi=D_{a g} \cdot B_{y}$ and we obtain $A g \subseteq B$. That $B \subseteq A g$ is shown in like manner, and hence $A_{x} \phi=g^{-1} \cdot A_{x} \cdot g$ for all $A_{x} \in K(S)$, and the result follows.

We do not know whether in the above Lemma it would suffice to simply assume the existence of constants based at each $x \in X$; we note however that our example 2 shows that it cannot in general be improved: to say something about the automorphisms of $S$ for example. But upon restricting our attention to transformation semigroups, we obtain a result with far-reaching implications: for its proof, we use an idea taken from Magill (1967); we shall use dom $\alpha$ to denote the domain of $\alpha$.

TheOREM 1. If $S$ is a subsemigroup of $\mathscr{P}_{X}$ covering $X$, then every automorphism of $S$ is inner.

Proof. Suppose $\phi \in \operatorname{Aut}(S)$. By Lemmas 2 and 3, there exists $g \in \mathscr{G}_{X}$ such that $A_{x} \phi=\mathrm{g}^{-1} \cdot A_{x} \cdot g$ for all $A_{x} \in K(S)$.

Now if $\alpha \in S$ and $x \in \operatorname{dom}(\alpha \phi)$, there exists $y \in X$ and $A_{x}, B_{y}$ in $K(S)$ with $x \in A, y \in B$ such that $B_{y} \phi=g^{-1} \cdot B_{y} \cdot g=A_{x}$. We therefore have

$$
A_{x} \cdot \alpha \phi=B_{y} \phi \cdot \alpha \phi=B_{y \alpha} \phi=g^{-1} B_{y x} g=g^{-1} B_{y} g \cdot g^{-1} \alpha g=A_{x} \cdot g^{-1} \alpha g
$$

Hence $\operatorname{dom}(\alpha \phi) \subseteq \operatorname{dom}\left(g^{-1} \alpha g\right)$. If now $x \in \operatorname{dom}\left(g^{-1} \alpha g\right)$, then there exists $y \in X$ such that $x g^{-1} \cdot \alpha=y g^{-1}$ and since $S$ covers $X$, there also exists $A_{x g^{-1}} \in K(S)$ with $x g^{-1} \in A$. Since $S$ is a semigroup, we then have $A_{y g-1}=A_{x g^{-1}} \cdot \alpha \in S$, and so under the automorphism $\phi$ we obtain

$$
A_{y g^{-1}} \phi=\left(A_{x g^{-1}} \cdot \alpha\right) \phi=A_{x g^{-1}} \phi \cdot \alpha \phi=g^{-1} A_{x g^{-1}} \cdot g \cdot \alpha \phi=g^{-1} A_{x} \cdot \alpha \phi .
$$

Hence $\operatorname{dom}\left(g^{-1} \alpha g\right) \subseteq \operatorname{dom}(\alpha \phi)$, and we deduce from the first equation displayed above that $\alpha \phi=g^{-1} \alpha g$ for all $\alpha \in S$.

Before proceeding, we note that there are quite trivial subsemigroups of $\mathscr{B}_{X}$ all of whose automorphisms are inner, but which do not cover $X$.

EXAMPLE 3. Fix $a, b \in X$, and put $\delta=\mathfrak{\imath}_{\{a b\}}$ and $S=\left\{1_{x}: x \neq a, b\right\} \cup\left\{\delta, b_{a}\right\}$ $\cup \square$. Then $S$ is a subsemigroup of $\mathscr{P}_{X}$ which does not cover $X$. If $\phi \in \operatorname{Aut}(S)$, then $\left(b_{a} \phi\right)^{2}=\square$ implies that $b_{a} \phi=b_{a}$. Suppose $i_{x} \phi=\delta$ for $x \neq a, b$. Then $b_{a}=b_{a} \cdot \delta=\left(b_{a} \cdot \mathfrak{l}_{x}\right) \phi$, and so $a=x$, a contradiction. Hence $\mathbf{t}_{x} \phi=\mathfrak{t}_{y}$ for some $y \neq a, b$; since this is also true of $\phi^{-1} \in \operatorname{Aut}(S)$ we obtain $\delta \phi=\delta$. Now define $g: X \rightarrow X$ by setting $x g=y$ if and only if $x \neq a, b$ and $\iota_{x} \phi=1_{y}$, and put $a g=a, b g=b$. Then clearly $g \in \mathscr{G}_{X}$ anf $\mathfrak{1}_{x} \phi=\mathfrak{\imath}_{y}=g^{-1} \cdot \mathfrak{\imath}_{x} \cdot g, \delta \phi=\delta=g^{-1} \delta g$, and $b_{a} \phi=b_{a}=g^{-1} b_{a} g$.

The above result will not in general suffice to describe the group $\operatorname{Aut}(S)$. On the other hand, for example, we know from Theorem 1 that every automorphism of $\mathscr{P}_{N}$ is inner and from Sutov (1961) that $\operatorname{Aut}\left(\mathscr{P}_{N}\right) \cong \mathscr{G}_{N}$. In the other 
extreme however, we now show that $\mathbf{t}_{N}$ is the only automorphism of the subsemigroup $S$ of $\mathscr{P}_{N}$ defined in Example 1.

For, if $\phi \in \operatorname{Aut}(S)$ and $\alpha_{n n} \phi=\alpha_{p q}$, then $q \leqq p$. Suppose $\alpha_{r s} \phi=\alpha_{q p}$. Then $\alpha_{q q}=\alpha_{q p} \cdot \alpha_{p q}=\left(\alpha_{r s} \alpha_{n n}\right) \phi$ and hence $s \leqq n$ and $\alpha_{r n} \phi=\alpha_{q q}$. We therefore have $\alpha_{q p}=\alpha_{q q} \cdot \alpha_{q p}=\left(\alpha_{r n} \cdot \alpha_{r s}\right) \phi$, and so $n \leqq r$. Hence $s \leqq r$; that is, both $\alpha_{r s}$ and $\alpha_{q p}$ are idempotent, and so $p=q$, and we have $\alpha_{n n} \phi=\alpha_{q q}$. Now suppose $1 \leqq k \leqq n$ and $\alpha_{n k} \phi=\alpha_{u v}$. Then

$$
\alpha_{q q}=\left(\alpha_{n k} \cdot \alpha_{n n}\right) \phi=\alpha_{u v} \cdot \alpha_{q q},
$$

and so $v \leqq q=u$. We can therefore define a mapping $\theta: B_{n} \rightarrow B_{q}$ by setting $k \theta=v$ if and only if $\alpha_{n k} \phi=\alpha_{q v}$. Since $\phi$ is injective, $\theta$ is also, and hence $n \leqq q$. In like manner, using $\phi^{-1} \in \operatorname{Aut}(S)$, we can obtain $q \leqq n$, and so for all $n \in N$, $\alpha_{n n} \phi=\alpha_{n n}$. Hence if $a, b \in N$ and $\alpha_{a b} \phi=\alpha_{x y}$, then

$$
\alpha_{x y}=\left(\alpha_{a a} \cdot \alpha_{a b}\right) \phi=\alpha_{a a} \alpha_{x y} \text { and } \alpha_{x y}=\left(\alpha_{a b} \cdot \alpha_{b b}\right) \phi=\alpha_{x y} \cdot \alpha_{b b}
$$

that is, $\alpha_{a y}=\alpha_{x b}$ and so $x=a$ and $y=b$. Therefore, every automorphism of $S$ is inner, and in fact $\operatorname{Aut}(S)=\left\{\mathfrak{t}_{N}\right\}$.

We have already noted (see Example 2 above) that Theorem 1 cannot readily be extended to subsemigroups of $\mathscr{B}_{X}$ : it can however be improved under the assumption of $\mathscr{G}_{X}$-normality. Of course, when this condition is present, we can, by Lemma 1, simply assume that our transformation semigroup contains at least one constant mapping.

THEOREM 2. If $S$ is a $\mathscr{G}_{X}$-normal subsemigroup of $\mathscr{P}_{X}$ containing a constant, then $\operatorname{Aut}(S) \cong \mathscr{G}_{X}$.

ProOF. To obtain an isomorphism between $\operatorname{Aut}(S)$ and $\mathscr{G}_{X}$, define $\theta_{g}: S \rightarrow S$ for $g \in \mathscr{G}_{X}$ by setting $\alpha \theta_{g}=g^{-1} \alpha g$ for all $\alpha \in S$, and $\operatorname{define} \Phi: \operatorname{Aut}(S) \rightarrow \mathscr{G}_{X}$ by putting $\theta_{g} \Phi=g$ for all $g \in \mathscr{G}_{X}$. It is then readily checked that $(\alpha \beta) \theta_{g}=\alpha \theta_{g} \cdot \beta \theta_{g}$ and that $\theta_{g}$ is injective. Moreover, $\left(g \alpha g^{-1}\right) \theta_{g}=\alpha$ and $g \alpha g^{-1}=\left(g^{-1}\right)^{-1} \alpha g^{-1} \in S$ implies that $\theta_{g}$ is surjective. Hence $\theta_{g} \in$ Aut $(S)$ and from Theorem 1 we know that every automorphism of $S$ is of this form. Hence $\Phi$ is well-defined, and it is easily verified that $\Phi$ is an isomorphism.

\section{Applications}

The next result is useful in proving the $\mathscr{G}_{X}$-normality of certain subsemigroup of $\mathscr{P}_{X}$ : its validity for $\mathscr{T}_{X}$ was noted in Mal'cev (1952); the generalisation we give was used by Sutov (1961).

LEMMA 4. If $\alpha, \beta \in \mathscr{P}_{X}$, then $\operatorname{rank}(\alpha \beta) \leqq \min (\operatorname{rank} \alpha$, rank $\beta)$.

It has been shown in Lyapin (1955), Magill (1967), Schreier (1936) that $\operatorname{Aut}\left(\mathscr{T}_{X}\right) \cong \mathscr{G}_{X}$. However Mal'cev (1952) extended this result by first showing that the ideals of $\mathscr{T}_{X}$ were of the form $T_{\xi}=\left\{\alpha \in \mathscr{T}_{X}:\right.$ rank $\left.\alpha<\xi\right\}$ where 
$1<\xi \leqq|X|^{\prime}$ : for an alternative account see Clifford and Preston (1967) and Sullivan (to appear; a). Since each $T_{\xi}$ is a subsemigroup of $\mathscr{P}_{X}$, contains $K\left(\mathscr{T}_{X}\right)=\left\{X_{a}: a \in X\right\}$, and by Lemma 4 , is $\mathscr{G}_{X \text {-normal, we can apply Theorems }}$ 1 and 2 to show that every automorphism of $T_{\xi}$ is inner and that $\operatorname{Aut}\left(T_{\xi}\right) \cong \mathscr{G}_{X}$ : a result first proved by Malcev (1952).

It was shown in Magill (1967) that $\operatorname{Aut}\left(\mathscr{P}_{X}\right) \cong \mathscr{G}_{X}$. However Sutov (1961) extended both this result and Malcev's work to $\mathscr{P}_{X}$, proving that every ideal of $\mathscr{P}_{X}$ is of the form $P_{\xi}=\left\{\alpha \in \mathscr{P}_{X}:\right.$ tank $\left.\alpha<\xi\right\}, 1 \leqq \xi \leqq|X|^{\prime}$ (see Sullivan (to appear; a) for an alternative account) and that when $\xi>1$, every automorphism of $P_{\xi}$ is inner and $\operatorname{Aut}\left(P_{\xi}\right) \cong \mathscr{G}_{X}$; this can now be deduced from Theorems 1 and 2 in a fashion analogous to that of $\mathscr{T}_{X}$ above.

Liber (1953) showed that the ideals of $\mathscr{I}_{X}$ were of the form $I_{\xi}=\mathscr{I}_{X} \cap P_{\xi}$, $1 \leqq \xi \leqq|X|^{\prime}$; see Sullivan (to appear; a) for an alternative account. Now each $I_{\xi}$ is a subsemigroup of $\mathscr{P}_{X}$ and contains $K\left(\mathscr{I}_{X}\right)=\mathscr{I}_{X} \cap K\left(\mathscr{P}_{X}\right)$. If $g \in \mathscr{G}_{X}$ and $\alpha \in I_{\xi}$, then clearly $g^{-1} \alpha g \in \mathscr{I}_{X}$ and so by Lemma $4, g^{-1} \alpha g \in I_{\xi}$. Hence Theorems 1 and 2 can again be applied to deduce that every automorphism of $I_{\xi}$ is inner and that $\operatorname{Aut}\left(I_{\xi}\right) \cong \mathscr{G}_{X}$, a result first proved by Liber (1953).

If $\alpha \in \mathscr{P}_{X}$, let def $\alpha=|X| X \alpha \mid$, and call this the defect of $\alpha$ (see Howie (1966)). Put $\mathscr{M}_{X}=\mathscr{T}_{X} \cap \mathscr{I}_{X}$ and suppose $|X|=p \geqq q \geqq \aleph_{0}$. Clifford and Preston (1961, 1967) called $M_{q}=\left\{\alpha \in \mathscr{M}_{X}: \operatorname{def} \alpha=q\right\}$ a Baer-Levi semigroup of type $(p, b)$ and clearly indicated the importance of the case $p=q$ in the general theory of right simple, right cancellative semigroups without idempotents. Now if $\alpha, \beta \in \mathscr{I}_{X}$ and de $\operatorname{def} \alpha=\operatorname{def} \beta=q$, then $X \alpha \beta \geqq X \beta$ implies that $\operatorname{def} \beta \leqq \operatorname{def}(\alpha \beta)$. In addition, $X \backslash X \alpha \beta=X \mid X \beta \cup X \beta \backslash X \alpha \beta$, and since $\beta$ is a 1-1 partial transformation, $|X \beta \backslash X \alpha \beta|$ $\leqq \operatorname{def} \alpha$. Hence $\operatorname{def}(\alpha \beta) \leqq q$; that is, $\left\{\alpha \in \mathscr{I}_{X} ; \operatorname{def} \alpha=q\right\}$ is a subsemigroup of $\mathscr{I}_{X}$, which we call a partial Baer-Levi semigroup of type $(p, q)$.

THEOREM 3. If $|X|=p \geqq \aleph_{0}$ and if $S$ is the partial Baer-Levi semigroup of type $(p, p)$, then every automorphism of $S$ is inner and $\operatorname{Aut}(S) \cong \mathscr{G}_{X}$.

Proof. If $i_{a}$ denotes the 1-1 partial transformation $\{(a, a)\}, a \in X$, then $\operatorname{def}\left(\mathfrak{l}_{a}\right)=|X| a|=| X \mid$, and so $S$ is a subsemigroup of $\mathscr{P}_{X}$ covering $X$. If $g \in \mathscr{G}_{X}$ and $\alpha \in S$, then

$$
|X| X g^{-1} \alpha g|=| X|X \alpha g|=|(X \mid X \alpha) g|=|X| X \alpha|=| X \mid
$$

and so $S$ is $\mathscr{G}_{X}$-normal. The result now follows from theorems 1 and 2.

Howie (1966) characterised the elements of the subsemigroup $\mathscr{E}_{X}$ of $\mathscr{T}_{X}$ generated by all the idempotents of $\mathscr{T}_{X}$. We now let $1<\xi \leqq|X|^{\prime}$ and let $E_{\xi}$ denote the set of all $\alpha \in \mathscr{E}_{X}$ such that rank $\alpha<\xi$ (cf. Sullivan (to appear; a)); by Lemma 4 , this is a subsemigroup of $\mathscr{E}_{X}$.

THEOREM 4. Every automorphism of $E_{\xi}, 1<\xi \leqq|X|^{\prime}$, is inner and $\operatorname{Aut}\left(E_{\xi}\right) \cong \mathscr{G}_{X}$. 
Proof. We have already noted that $E_{\xi}$ is a subsemigroup of $\mathscr{P}_{X}$ and since $K\left(\mathscr{T}_{X}\right) \subseteq E_{\xi}$ for $\xi>1$, it in fact covers $X$.

If $g \in \mathscr{G}_{X}$ and $\alpha=\delta_{1} \cdots \delta_{r} \in E_{\xi}, \delta_{i}{ }^{2}=\delta_{i}, i=1, \cdots, r$, then $g^{-1} \alpha \mathrm{g}=g^{-1} \delta_{1} g \cdot$ $\cdots g^{-1} \delta_{r} g$, and for each $i=1, \cdots, r, g^{-1} \delta_{i} g \in \mathscr{E}_{X}$. In addition, by Lemma 4, $\operatorname{rank}\left(g^{-1} \alpha g\right)<\xi$. The result now follows from Theorems 1 and 2 .

Before continuing we note that a similar result (with an identical proof) can be given for the subsemigroup of $\mathscr{P}_{X}$ generated by all the idempotents of $\mathscr{P}_{X}$ : the elements of this semigroup, $X$ finite, were characterised by Sullivan (1969) and in the general case by Evseev and Podran (1970).

If $\alpha \in \mathscr{P}_{X}$, we put $S(\alpha)=\{x \in \operatorname{dom} \alpha: x \alpha \neq x\}$ and $s(\alpha)=|S(\alpha)|$ and call this the shift of $\alpha$ (see Howie (1966)). Note that since $S(\square)=\square$, we can meaningfully define $s(\square)$ to be zero. For each cardinal $\xi$, we now put $W(X, \xi)=\left\{\alpha \in \mathscr{P}_{X}\right.$ : $s(\alpha)<\xi\}$. If $X$ is finite, $W(X, \xi)$ need not be a semigroup.

EXAmple 4. Let $X=\{1, \cdots, n\}$ and suppose $2 \leqq \xi=r+1 \leqq n$. Define $\alpha, \beta \in \mathscr{P}_{X}$ by setting

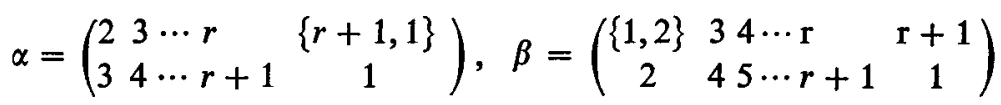

Then $s(\alpha)=s(\beta)=r$, but

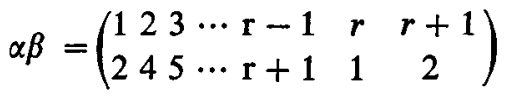

has shift $r+1$.

However, the following well-known result shows that if $\xi \geqq \aleph_{0}$, then $W(X, \xi)$ is a subsemigroup of $\mathscr{P}_{X}$.

Lemma 5. If $\alpha, \beta \in \mathscr{P}_{X}$, then $S(\alpha \beta) \subseteq S(\alpha) \cup S(\beta)$.

Sutov described the ideals and automorphisms of $W\left(X, \aleph_{0}\right)$. We extend his results to $W(X, \xi)$ : for a characterisation of the ideals of $W(X, \xi)$ see Sullivan (to appear; a).

THEOREM 5. If $\xi \geqq \aleph_{0}$, then every automorphism of $W(X, \xi)$ is inner and $\operatorname{Aut}(W(X, \xi)) \cong \mathscr{G}_{X}$.

Proof. Clearly $K(W(X, \xi))=\left\{A_{x} \in \mathscr{P}_{X}:|A|<\xi\right\}$. Hence $W(X, \xi)$ is a subsemigroup of $\mathscr{P}_{X}$ covering $X$ and so Theorem 1 gives the first part of the result. If $g \in \mathscr{G}_{X}$ and $\alpha \in W(X, \xi)$ and $x \in S\left(g^{-1} \alpha g\right)$, then $x g^{-1} \alpha g \neq x$ and so $x g^{-1} \alpha \neq x g^{-1}$; that is, $x g^{-1} \in S(\alpha)$. Hence $S\left(g^{-1} \alpha g\right) \subseteq S(\alpha) g$ and we have $g^{-1} \alpha g \in W(X, \xi)$ and $W(X, \xi)$ is $\mathscr{G}_{X}$-normal; an application of Theorem 2 now completes the proof.

Since $\mathscr{P}_{X}=W(X, \xi)$ when $X$ is finite and $\xi=\aleph_{0}$, and when $X$ is infinite and $\xi=|X|^{\prime}$, the above result generalises Sutov's work outlined in the third 
paragraph of this section. Finally, we note that a result similar to that of Theorem 5 holds for $I(W, \xi)=\mathscr{I}_{X} \cap W(X, \xi)$, and that this fact can be regarded as an extension of the work of Scott (1964) on determining the automorphism groups of the normal subgroups of $\mathscr{G}_{X}$.

\section{References}

A. H. Clifford and G. B. Preston (1961, 1967), The Algebraic Theory of Semigroups, (Math. Surveys, No. 7, Amer. Math. Soc., Providence, RI, Volume, 11961 : Volume 2, 1967).

A. E. Evseev and N. E. Podran (1970), 'Semigroups of transformations generated by idempotents with given projection characteristics', Izv. Vyss. Ucebn. Zaved. Mat. 12 (103), 30-36.

L. M. Gluskin (1959), 'Ideals of semigroups of transformations', Mat. Sb. (NS) 47 (89), 111-130.

J. M. Howie (1966), 'The subsemigroup generated by the idempotents of a full transformation semigroup', J. London Math. Soc. 41, 707-716.

A. E. Liber (1953), 'On symmetric generalised groups', Mat. Sb. (NS) 33 (75), 531-544.

E. S. Lyapin (1955), 'Abstract characterisation of certain semigroups of transformations', Lenningrad Gos. Ped. Inst. Ucen. Zap. 103, 5-29.

K. D. Magill, Jnr. (1966), 'Automorphisms of the semigroup of all relations on a set', Canad. Math. Bull. 9, 73-77.

K. D. Magill, Jnr. (1967), 'Semigroup Structures for Families of Functions, I. Some Homomorphism Theorems', J. Austral. Math. Soc. 7, 81-107.

A. I. Malcev (1952), 'Symmetric Groupoids', Mat. Sb. (NS) 31 (73), 136-151.

O. Schreicr (1936), 'Utber Abbildungen einer abstrakten Menge auf ihre Teilmengen', Fund. Math. 28, 261-264.

W. Scott (1964), Group Theory, (Prentice Hall, 1964).

R. P. Sullivan (1969), A study in the theory of transformation semigroups, Ph. D. Dissertation, (Monash University August, 1969.)

R. P. Sullivan (to appear), 'Automorphisms of relation semigroups'.

R. P. Sullivan (to appear; a), 'Ideals in transformation semigroups'.

E. G. Sutov (1961), 'Homomorphisms of the semigroup of all partial transformations', Izeves. Vyss. Ucebn. Zaved. Mat. 3 (22), 177-184.

E. G. Sutov (1961a), 'On semigroups of almost identical transformations,' Dokl. Akad. Nauk. SSSR 134 (1960), 292-295; translated as Soviet Math. Dokl., 1, 1080-1083.

University of Western Australia

Nedlands, W. A. 6009 